\title{
Effects of interface roughness on the cohesive strength of self-assembled monolayers
}

Zhang, Chen, czhang49@illinois.edu; Awasthi, Amnaya; Grady, Meg; Sottos, Nancy; Geubelle, Philippe, University of Illinois, United States

\begin{abstract}
Self-assembled monolayers (SAM) are aggregates of small molecular chains that have the property to form highly ordered assemblies. The presence of terminal groups on the constituent chains makes them excellent contenders of molecular level tailoring. Molecular Dynamics (MD) simulations and experimental observations of spallation have shown that cohesive strength of SAM-enriched molecular interfaces is strongly dependent on the choice of terminal groups. Although the MD results of perfectly ordered atomistic surfaces show the same qualitative trend as the experiments, yet they overpredict the interfacial cohesive strengths by factors of 30-50. Results from AFM studies have revealed that the roughness of these interfaces is the same order $(\sim 1 \mathrm{~nm})$ as the range of atomistic interactions. Hence, surface roughness is a key contributor in significantly reducing interfacial cohesive strength in these systems. In this research, a continuum level numerical study is performed to investigate the influence of surface roughness on cohesive strength of the interface between SAM and a thin film of gold. We approximate the film as a deformable continuum interacting with a rough substrate of SAM represented by a harmonic function. Using the cohesive law predicted by MD, quasi-static separation tests are performed to evaluate the effective traction separation characteristics for the rough SAM-gold interface. The analysis is effective in demonstrating that the incorporation of roughness reduces the cohesive strength of the interface by up to $90 \%$. Additionally, it is also observed that the gold film adopts unique separation attributes based on roughness parameters and material properties.
\end{abstract}

